\title{
H7N9-Vogelgrippe in China
}

Influenzaviren der Gruppe A/H7 sind hauptsächlich an Vögel adaptiert, jedoch gab es in der Vergangenheit einige einzelne Fälle, bei denen auch Menschen an diesen typischen Vogelgrippeviren erkrankten. Im März dieses Jahres wurde in China nun erstmals ein humaner Fall gemeldet, der durch eine Variante des A/H7N9-Influenzavirus hervorgerufen wurde. Innerhalb der nächsten 11 Wochen erkrankten mindestens 130 weitere Menschen. Bei den meisten der gemeldeten Fälle nahm die Erkrankung einen schweren Verlauf. Bis Mitte Mai waren 35 Personen an den Folgen der Infektion verstorben.

Zunächst waren die Fälle auf den Osten des Landes beschränkt, betroffen waren ausschließlich Shanghai und die angrenzenden Provinzen Anhui, Jiangsu und Zhejiang. Seit Mitte April wurden aber auch aus weiteren östlichen, zentralen und nördlichen Regionen Chinas erste Fälle gemeldet. Ein Fall wurde nach Taiwan importiert.
Die Übertragungswege der Krankheit geben nach wie vor Rätsel auf: Es gibt bisher keine Hinweise darauf, dass das Virus von Mensch zu Mensch übertragen werden kann. Jedoch scheinen auch etwa ein Viertel der Erkrankten keinerlei Kontakt zu Vögeln gehabt zu haben.

Interessanterweise handelt es sich bei ungewöhnlich vielen Betroffenen um ältere Männer (71\% männlich, 63\% über 60 Jahre) - ganz entgegen dem Bevölkerungsdurchschnitt. Eigentlich dominieren in China die jungen und mittleren Altersgruppen und bei den Älteren gibt es deutlich mehr Frauen als Männer.

Seit Anfang Mai scheint die Zahl der Neuinfektionen zu sinken. Ob dies auf ein effizientes Krisenmanagement der chinesischen Regierung (etwa durch Schließung der Geflügelmärkte) zurückzuführen ist, oder aber saisonal bedingt ist, lässt sich derzeit noch nicht abschätzen. Möglicherweise spielen beide Faktoren eine Rolle.

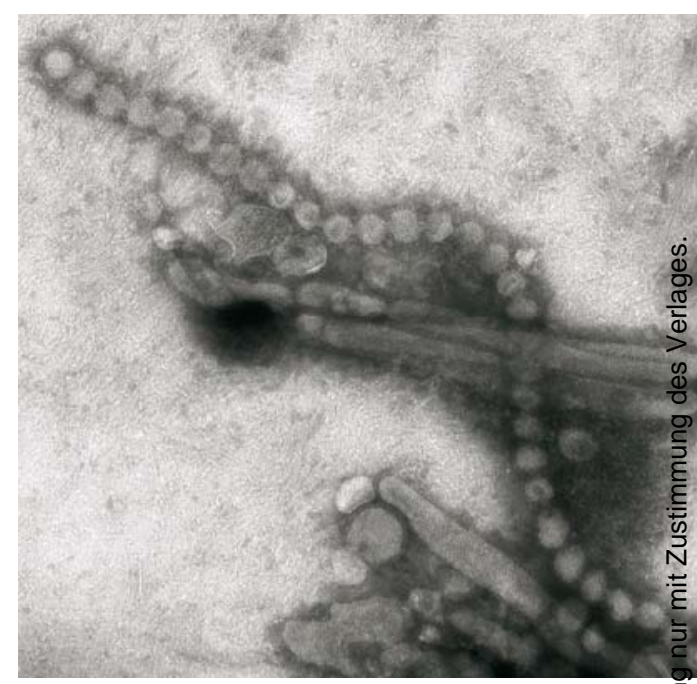

Die neue H7N9-Variante, aufgenommen mit dem Transmissionselektronenmikroskop (TEM). Quelle: Centers for Disease Control and Prevention (CDC)/Cynthia S. Goldsmith and Thomas Rowe

Dr. Raymund Lösch und Dipl. Biol. Unn Klare, Bad Doberan

Quellen: promed, WHO

\section{Poliomyelitis in Ostafrika wieder aufgetreten}

Mitte April wurde zunächst aus der somalischen Region Banadir ein Fall von Kinderlähmung gemeldet. Einen Monat später erkrankte auch im kenianischen Dadaab ein Kind an Poliomyelitis. Bei 2 gesunden Kontaktpersonen des kenianischen Patienten konnten ebenfalls Polioviren nachgewiesen werden.

Der letzte kenianische Poliofall lag 2 Jahre, der letzte Fall aus Somalia gar 6 Jahre zurück. Dieses Wiederauftauchen der Kinderlähmung in Ländern, die jahrelang frei von Poliomyelitisfällen waren, ist ein trauriger Rückschlag in der Bekämpfung dieser Krankheit. Und kommt zu einem Zeitpunkt, an dem eigentlich die Hoffnung bestand, die Krankheit endlich ausrotten zu können. Nachdem Indien seit Januar 2011 keine neuen Infektionen mit Polio-Wildviren mehr gemeldet hatte, gibt es derzeit nur noch 3 Länder, in denen die Kinderlähmung endemisch ist. Und in allen dreien wurden dieses Jahr bisher weniger Fälle gemeldet als im Vergleichszeitraum des Vorjahres: Nigeria (dieses Jahr 22, Vorjahr 30 Fälle), Pakistan (dieses Jahr 8, Vorjahr 16 Fälle)

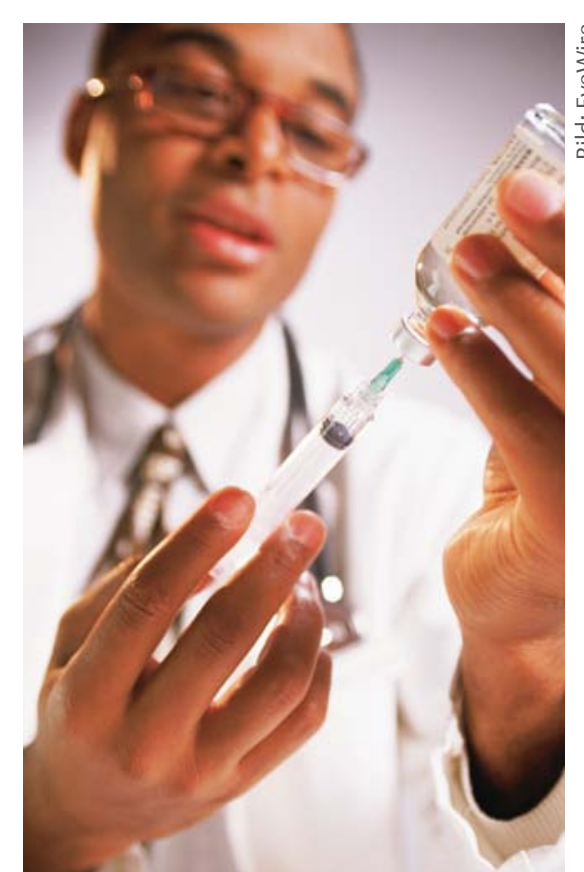

und Afghanistan (dieses Jahr 2, Vorjahr 6 Fälle).

Aber trotz der Fortschritte in diesen 3 Ländern gibt es auch dort Grund zur Besorgnis: Nach wie vor werden Gerüchte propagiert, die Impfstoffe würden unfruchtbar machen, um so die muslimische Bevölkerung zu schwächen, oder die CIA nutze die Impfkampagnen, um die Bevölkerung auszuspionieren. Vielfach werden die Impfungen aus religiösen Gründen abgelehnt. Diese Überzeugungen gipfelten in den vergangenen Monaten in mehreren Anschlägen auf medizinisches Personal, das die Durchführung von Impfkampagnen vorbereitete. In Nigeria starben bei 2 Angriffen Anfang Februar insgesamt 9 Menschen. Seit Ende letzten Jahres wurden in Pakistan 7 Personen getötet.

Dr. Raymund Lösch und Dipl. Biol. Unn Klare, Bad Doberan

Quelle: promed 


\section{Neues Coronavirus im Nahen Osten}

Mitte September des vergangenen Jahres wurde ein neues Coronavirus beschrieben (MERS-CoV= Middle East respiratory syndrome coronavirus), das anscheinend schwere respiratorische Krankheiten beim Menschen hervorrufen kann (wir berichteten). Seither wurden insgesamt 49 Fälle nachgewiesen.

Die Infektionen erfolgten fast ausschließlich im Nahen Osten, die meisten in Saudi-Arabien (36 Infektionen, darunter 18 mit Todesfolge). In Katar infizierten sich 2 Personen, in Jordanien verstarben 2 Menschen an den Folgen der Infektion und eine Person, die sich vermutlich in den Vereinigten Arabischen Emiraten infi-

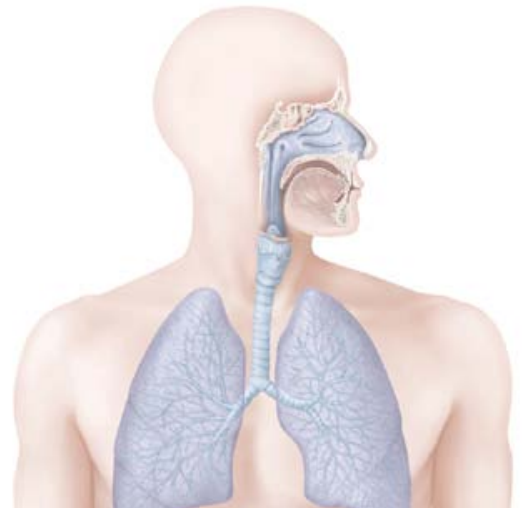

Coronaviren rufen verschiedene und unterschiedlich stark ausgeprägte Erkrankungen der Atemwege hervor.

Quelle: Thieme Verlagsgruppe/Voll ziert hatte, erlag ebenfalls der Krankheit. Infektionen meldeten außerdem auch Großbritannien (3 Fälle, darunter 2 mit Todesfolge), Tunesien (3 Fälle, ein Todesopfer) und Frankreich (2 Fälle, ein Todesopfer), wobei sich die Indexfälle kurz vor Krankheitsausbruch in Saudi-Arabien beziehungsweise in den Vereinigten Arabischen Emiraten aufgehalten hatten.

Seit Herbst letzten Jahres waren zunächst nur sporadische Einzelfälle oder kleinere Cluster des neuen Coronavirus gemeldet worden. Ende April 2013 begann in einem Krankenhaus im Osten Saudi-Arabiens ein Ausbruch, bei dem innerhalb von etwa 3 Wochen 25 Fälle identifiziert wurden - etwa die Hälfte der bisher weltweit bestätigten Fälle.

Wie das neue Virus übertragen wird, ist bisher nicht bekannt. Es scheint jedoch zumindest eingeschränkt von Mensch zu Mensch übertragbar zu sein. So wurden bisher 6 Cluster festgestellt. Während in 3 der Fälle auch eine Ansteckung bei einer gemeinsamen Infektionsquelle möglich ist, scheint zumindest bei dem britischen, dem tunesischen und dem französischen Cluster eindeutig eine Mensch-zuMensch-Übertragung aufgetreten zu sein: Nur jeweils einer der Betroffenen hatte sich vor Krankheitsbeginn im Nahen Osten aufgehalten. Die anderen Erkrankten hatten sich offensichtlich bei dem Reisen- den nach dessen Rückkehr nach Europa infiziert. Die weltweit geringe Zahl der bisher bestätigten Fälle deutet jedoch darauf hin, dass das Virus nicht leicht unter Menschen weiter verbreitet wird. Die WHO geht derzeit davon aus, dass ein längerer und sehr enger Kontakt für eine Übertragung erforderlich ist.

Als mögliches Virusreservoir konnten verschiedene, weit verbreitete Fledermausarten identifiziert werden. Bei ihnen wurden Coronaviren nachgewiesen, die den für die humanen Fälle verantwortlichen stark ähneln. Ob Fledermäuse die direkte Infektionsquelle sind, ist bisher noch nicht geklärt. Möglicherweise könnte - ähnlich wie beim Nipah-Virus durch Fledermäuse kontaminierter Dattelsaft eine Rolle spielen. In einem Fall wurde darüber hinaus vermutet, dass die Ansteckung durch Kontakt zu einem erkrankten Kamel erfolgt sein könnte.

Symptome der Erkrankung sind Fieber, Husten und andere respiratorischen Symptome. Coronaviren können von einem einfachen Schnupfen bis hin zu SARS (Severe Acute Respiratory Syndrome) verschiedene und unterschiedlich stark ausgeprägte Erkrankungen der Atemwege bei Mensch und Tier hervorrufen.

Dr. Raymund Lösch und Dipl. Biol. Unn Klare, Bad Doberan

Quellen: promed, WHO

\section{Griechenland nicht mehr frei von Tollwut}

Mitte Oktober 2012 bedrohte ein aggressiver Rotfuchs die Bewohner eines Dorfes im Westen der Region Makedonien - wie sich später herausstellte, handelte es sich um den ersten nachgewiesenen Tollwutfall Griechenlands seit Jahren. In den folgenden 6 Monaten wurde die Krankheit bei 13 weiteren Füchsen sowie 2 Hütehunden und einer Katze diagnostiziert. Alle Fälle hatten sich im Norden oder im Zentrum des Landes ereignet. Insgesamt mussten 104 Menschen nach Kontakt zu den Tieren behandelt werden.

Der letzte humane Tollwutfall Griechenlands liegt 43 Jahre zurück. Das Land war im Jahr 1987 als tollwutfrei erklärt worden. Damit steht Griechenland jedoch im Balkan und den angrenzenden Gebieten relativ allein da. Sowohl in Griechenlands Nachbarstaaten Bulgarien und der Türkei als auch in Ungarn, Serbien und Rumänien ist die Tollwut nach wie vor etabliert und scheint sich aus diesen Gebieten südund westwärts auszubreiten.

Dr. Raymund Lösch und Dipl. Biol. Unn Klare, Bad Doberan

Quellen: promed; Tsiodras S, Dougas G, Baka A et al. Re-emergence of animal rabies in northern Greece and subsequent human exposure, October 2012 March 2013. Euro Surveill 2013; 18: pii: 20474

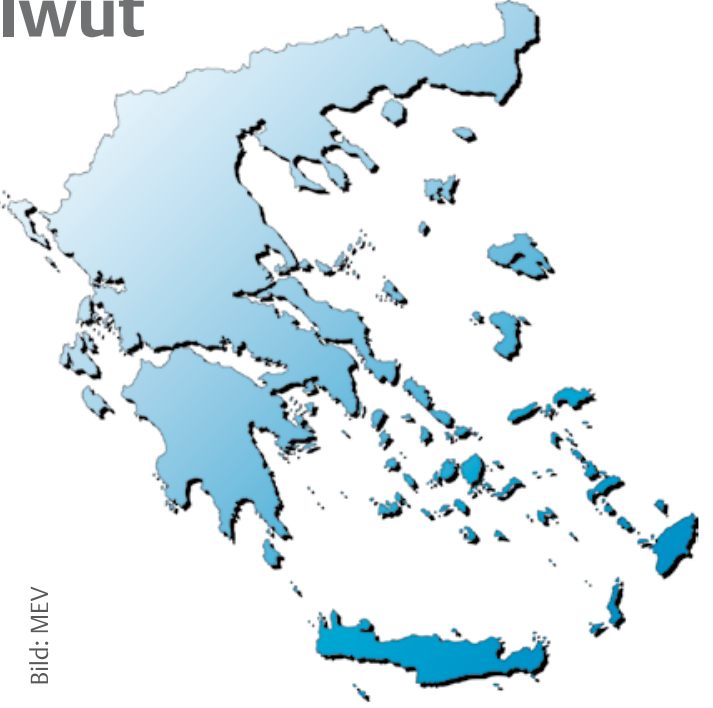




\section{Aktuelles kurz notiert}

Chikungunyafieber in Papua-Neuguinea Auf Papua-Neuguinea wurden im Jahr 2012 erstmals Fälle von Chikungunyafieber gemeldet. Seither scheint sich die Infektionskrankheit weiter auszubreiten. Die ersten Fälle waren in der Umgebung der Stadt Vanimo an der Grenze zu WestPapua (Indonesien) aufgetreten. Mittlerweile sind vermutlich 8 Provinzen betroffen. Die Zahl der Erkrankten wird mit

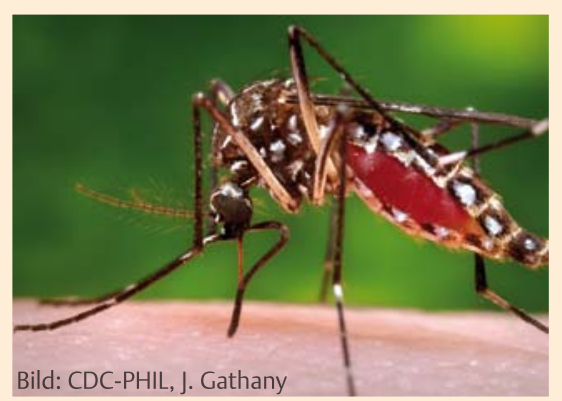

etwa 3000 angegeben. Das Auftreten der Krankheit in Papua-Neuguinea ist nicht überraschend, da Aedes-Mücken, die Vektoren dieses Fiebers, hier weit verbreitet sind.

\section{Lebensmittelvergiftungen}

in ägyptischer Universität

Ende April erkrankten 161 Studenten der al-Azhar-Universität in Kairo an einer Lebensmittelvergiftung. Die Ursache war schlechter Thunfisch in der Cafeteria auf dem Campus. An der gleichen Universität mussten knapp 4 Wochen zuvor bereits mehr als 500 Studenten

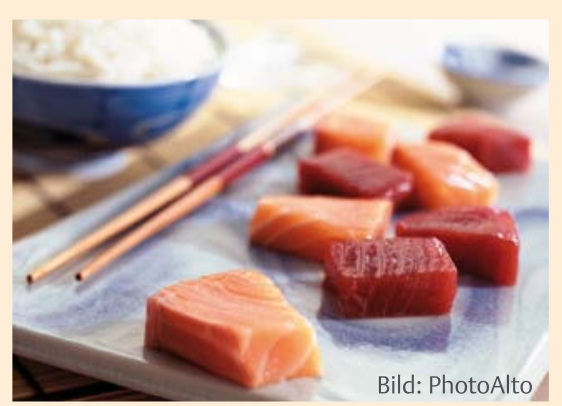

aufgrund einer Magen-Darm-Erkrankung hospitalisiert werden. Lebensmittelvergiftungen sind anscheinend in den Universitäten Ägyptens keine Seltenheit, da hier oft grundlegende Hygiene- standards nicht eingehalten werden. Die Zahl der dieses Jahr bereits erkrankten Studenten nimmt jedoch ein bedenkliches Ausmaß an.

\section{Staphylokokkeninfektionen auf Réunion}

Seit Mitte Februar erkrankten anscheinend mehrere Hundert Menschen auf Réunion an Infektionen mit dem Bakterium Staphylococcus aureus durch Kontakt zu Meerwasser am Strand des Ortes L'Étang-Salé. Der Strand ist ein beliebter Ort für Surfer. Unter den Betroffenen waren viele Wassersportler, aber auch zahlreiche Fischer. Sie litten unter schmerzhaften Hautentzündun-

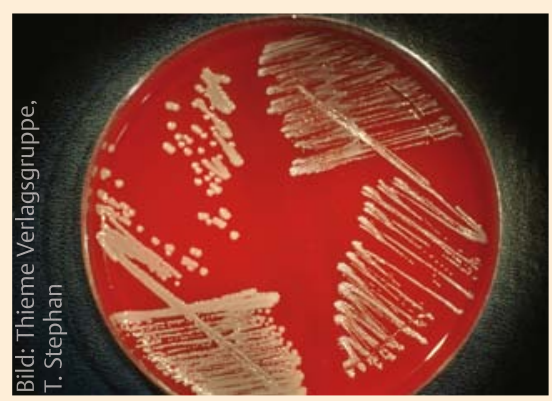

gen. Kontakt zu Meerwasser ist kein typischer Risikofaktor für Staphylokokkeninfektionen. Vereinzelt wurden aber ähnliche Ausbrüche auch in der Vergangenheit schon beschrieben. Kurz vor dem Auftreten der ersten Fälle war ein Wirbelsturm über Réunion hinweggefegt und hatte zur Verunreinigung der Küstengewässer geführt. Erste Wasserproben von dem betroffenen Strand hatten jedoch keine Auffälligkeiten ergeben.

Östliche Pferdeenzephalomyelitis in den USA

Ungewöhnlich früh im Jahr wurde Mitte März aus Florida der erste diesjährige US-amerikanische Fall von Östlicher Pferdeenzephalomyelitis gemeldet. Der in einigen Regionen des Landes doch sehr milde Winter ist vermutlich für diese frühe Infektion verantwortlich. Es handelt sich um eine seltene Erkrankung beim Menschen. Jährlich treten in den USA in der Regel lediglich 5-10 Fälle auf.

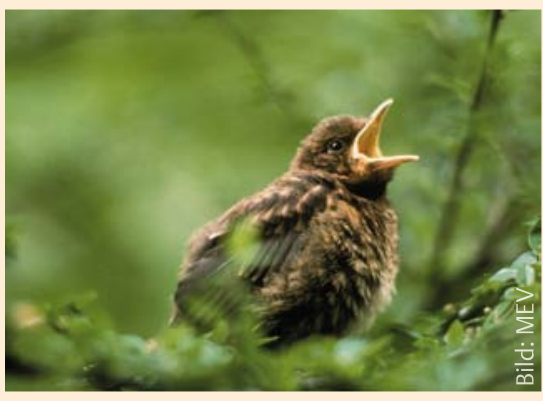

Psittakose in Schweden

Seit Beginn des Jahres erkrankten in Schweden bereits 25 Menschen an der Psittakose. Eine Person verstarb an den Folgen der Infektion. Normalerweise werden landesweit im Verlauf eines ganzen Jahres lediglich 5-10 Fälle gemeldet. Fast alle diesjährigen Infektionen waren in den Regionen Skane und Kronberg im Süden des Landes erfolgt. Die meisten Erkrankten hatten vor dem Auftreten der ersten Symptome Kontakt zu Federn wildlebender Vögel im eigenen Garten.

Krim-Kongo-Fieber in der Türkei

Ende April wurde der erste diesjährige Todesfall aufgrund des hämorrhagischen Krim-Kongo-Fiebers aus der Türkei gemeldet. Darüber, wie viele Personen erkrankten, liegen derzeit keine Informationen vor. Die Türkei ist das Land, aus dem weltweit die meisten Fälle gemeldet werden (was aber auch an der Effektivi-

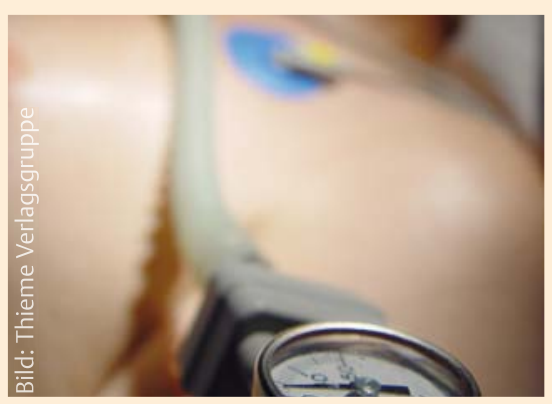

tät des Surveillance-Systems liegen mag). Seit dem Jahr 2002 verstarben hier insgesamt 175 Menschen. Jährlich erkranken derzeit etwa 1000 Personen.

Dr. Raymund Lösch und Dipl. Biol. Unn Klare, Bad Doberan

Quelle: promed 